\title{
Analysis and design of a micro electromagnetic vibration energy harvester
}

\author{
Xiongshi Wang ${ }^{1, a}$, Binzhen Zhang ${ }^{1, b}$, Junping Duan ${ }^{1, c}$, Suping $\mathrm{Xu}^{1, \mathrm{~d}}$ \\ ${ }^{1}$ North University of China Science and Technology on Electronic Test \&Measurement Laboratory, \\ Taiyuan 030051, China.

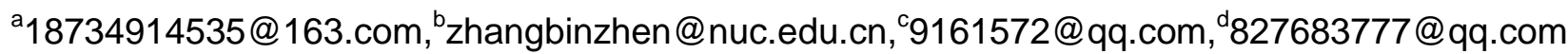

Keywords: Micro Energy Harvester, Low Frequency, Modal Analysis, Fatigue Analysis.

\begin{abstract}
At present the commonly used energy harvesters have characteristics of low harvester efficiency and short life. This paper presents a micro-energy harvester, the microstructure and collection system are combined to improve the energy efficiency, and optimize the structural parameters to realize the prolongation of life. Firstly, the general principle of electromagnetic energy harvester design was introduced. Static and modal simulation for single-beam, dual-beam, three beams, and four beams are analyzed and compared using finite element simulation software ANSYS. Further study of the combined effect of the length of the four-beam energy collector beams length, width and height on the maximum deformation and natural frequencies, and optimize the structure. Finally, based on nCode DesignLife, the result of the life of the micro energy harvester under high acceleration times up $3.092 \times 10^{18}$.
\end{abstract}

\section{Introduction}

Over the last decade, new sensor technology driven by MEMS technology is developing constantly, and different kinds of sensors is emerging [1]. At the same time the power supply of sensor has become an important factor limiting its development, particularly in some special cases, the power cord has become the most fatal flaw of the power cable. Thus armed miniature equipment, handheld devices and micro independent Microsystems implantable medical devices, etc., based on the power cable transmission means helpless, and the continuous development of wireless power has opened another window for people, so people favor and pay attention to it [2, 4].

According to the energy sources, the energy harvester can be divided into three kinds, which are electromagnetic radiation energy harvester, heat energy harvester and vibration mechanical energy harvester [5]. Wherein the solar radiation is a form of electromagnetic radiation in the current development of the most mature, but greatly limited by sunlight. For example cloudy, or even some places like deep sea or mine. Another form is radio-frequency radiation, and Nicola has successfully achieved the collection of smart mobile phone radiation energy, which is the world's leading level in this aspect. Firstly it designed an antenna for collecting energy generated by the mobile networks and WIFI network, then the DC voltage connected on the wireless device charging port is converted into standard voltage devices which meet the needs of charging device by the power management circuitry which converts RF energy into DC with the highest efficiency. Using the mobile network or WIFI is obvious, according to Nikola laboratory evaluation, when downloading a file, the phone's battery life can increase about 30\%. Pity is currently only applicable for two versions, iPhone6 version and Galaxy S6 version. Also, for the recent scientists in Holland have developed a miniature sensor [6], it is possible to exchange information by means of radio wave energy, only $2 \mathrm{~mm}^{2}$ size and weight of $1.6 \mathrm{mg}$. Heat collection is based on the thermoelectric effect of semiconductor material, there are two harsh conditions, one is heat source, the other is large temperature difference. Otherwise, the generating efficiency will be greatly reduced, it is currently required to overcome the problem of heat collector that high temperatures and large temperature difference will have a serious impact on the performance and life of micro-nano device. [7, 9]. 


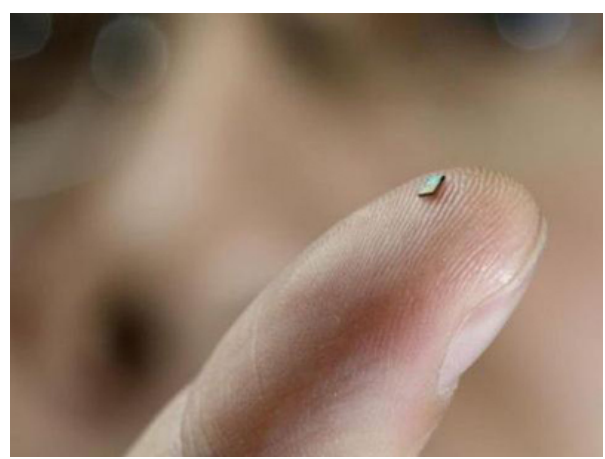

Fig. 1 A miniature sensor developed by scientists in Holland [6]

Mechanical vibration energy harvester that is obtained from external vibration environments transformed into electrical energy. According to its working principle, divided into three forms: electrostatic, piezoelectric and electromagnetic. Electrostatic type is to collect energy using variable capacitor plate spacing or in the relative area, but we need to impose a polarization voltage before it works. In addition, the general processing technology is also more complex. Piezoelectric Energy Harvesting is the use of piezoelectric effect piezoelectric material to generate electricity, no external power supply, the disadvantage is difficult to use piezoelectric material micromachining process. Electromagnetic energy harvester is based on Faraday's law of electromagnetic induction, suitable for medium and low frequency vibration environment, when the permanent magnet and the coil have relative motion, in the closed loop it will arise opposite current $[10,11]$.

\section{Principle Description}

Figure 2 shows a schematic diagram of a miniature vibrating energy harvester.

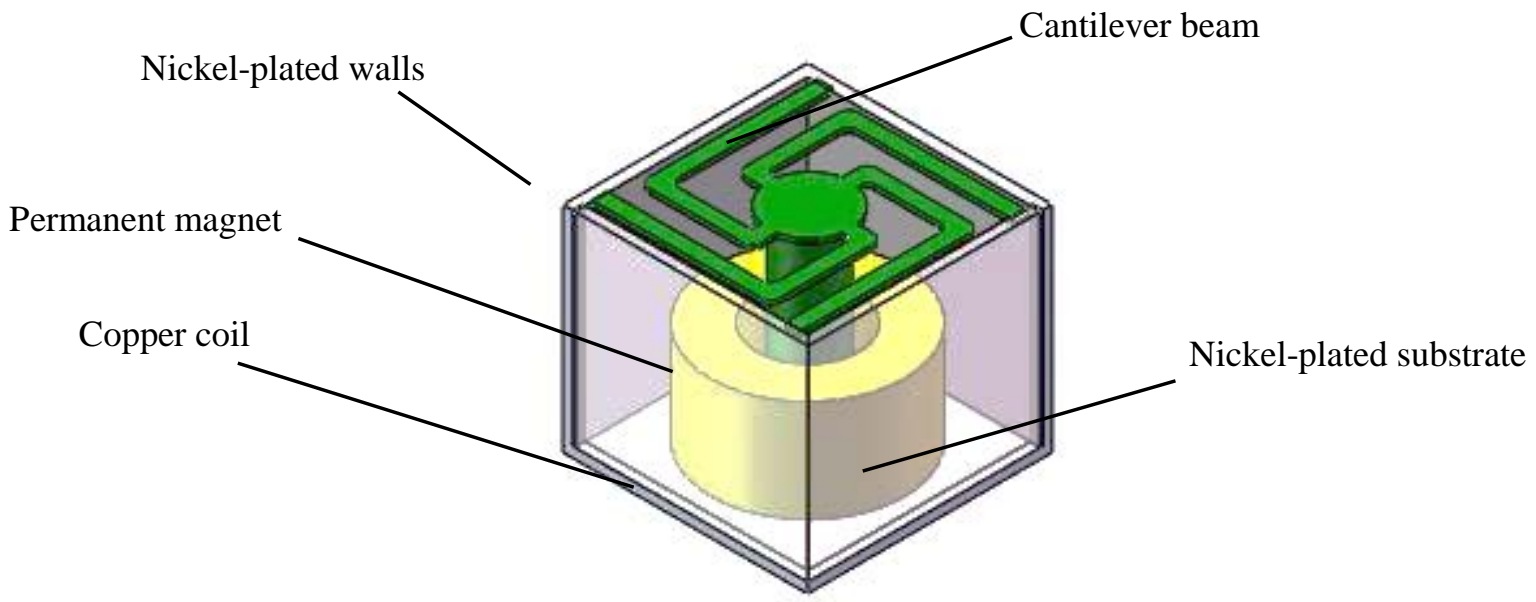

Fig. 2 Schematic diagram of micro vibration energy harvester

According to Faraday's law of electromagnetic induction, when the magnetic flux changes, it will produce a of the induced electromotive force in the opposite direction hinders in the magnetic circuit and slows down the change, the core idea of which is that most electromagnetic energy harvester design and theory basis. Therefore, our design model has the following two parts: nickel-plated wall and nickel-plated substrate constitute the complete magnetic circuit, cantilever beam, copper coil, permanent magnet to form the magnetic field of the relative magnetic circuit.

\section{Simulation and Analysis of electromagnetic vibration energy collector with different beam structure}

For electromagnetic vibratory energy harvester, we use FEM software ANSYS to research and analysis. In the preparation of micro technology based on UV-LIGA, the energy collector thickness relative to the length is negligible, however, taking into account the impact of thickness of on the 
beam maximum deformation and resonant frequency too large, a detailed three-dimensional structure study was carried out in this article.

Considering the specific MEMS process used, this paper analyzes the structure of four kinds of beam energy collector: single-beam, dual-beam, three beam and four beams, as shown in Figure 3, a single beam structure diagram shown in Figure 4.
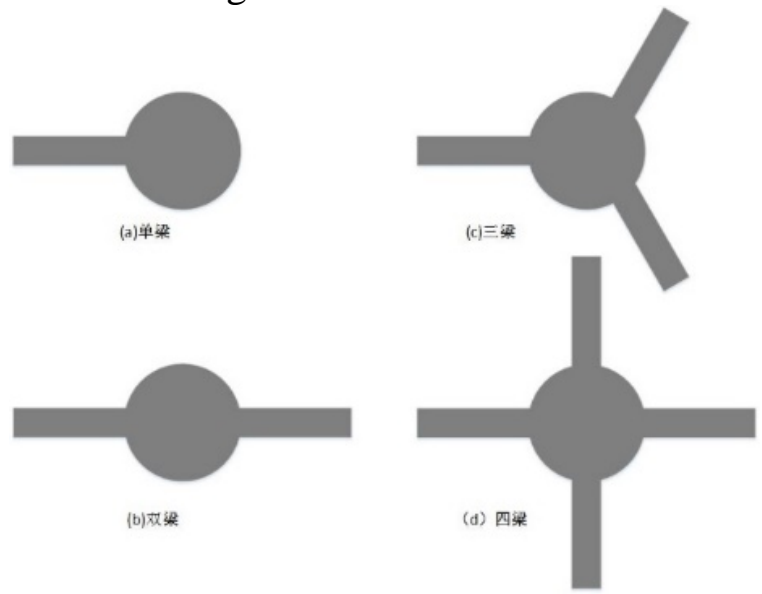

Fig. 3 The structure of four kinds of beam energy collector

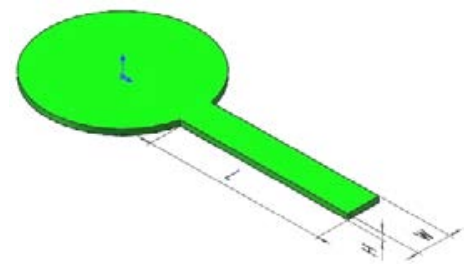

Fig. 4 Single beam structure model

To study their maximum deformation and stress conditions, we carried out static analysis, applying $0.1 \mathrm{~N}$ uniform load across the beam. Length setting each energy harvester structure is $2000 \mu \mathrm{m}$, width of $500 \mu \mathrm{m}$, the thickness is set to $52.5 \mu \mathrm{m}$. Beam is made of nickel, its elastic modulus is $2.1 \mathrm{E} 11 \mathrm{~Pa}$, Poisson's ratio is 0.31 , a yield strength is $100-600 \mathrm{MPa}$, a tensile strength is $310-670 \mathrm{MPa}$, a density is $8800 \mathrm{Kg} \mathrm{m}^{-3}$. Center disk diameter is $3800 \mu \mathrm{m}$, with a diameter of $1800 \mu \mathrm{m}$, $2000 \mu \mathrm{m}$ height cylindrical $\mathrm{NdFeB}$ below, its density is $6550 \mathrm{Kg} \mathrm{m}^{-3}$. The results of stress and maximum deformation energy harvester beams as shown in Table 1.

Tab. 1 The analysis results of different shapes of the energy harvester

\begin{tabular}{|c|c|c|c|c|}
\hline \multirow{2}{*}{$\begin{array}{c}\text { Different } \\
\text { Beam }\end{array}$} & \multicolumn{2}{|c|}{ Static Analysis } & \multicolumn{2}{c|}{ Modal Analysis } \\
\cline { 2 - 5 } Structure & $\begin{array}{c}\text { Stress } \\
(\mathrm{Pa})\end{array}$ & $\begin{array}{c}\text { Maximum } \\
\text { Deformation }(\mu \mathrm{m})\end{array}$ & $\begin{array}{c}\text { First } \\
\text { Modal }(\mathrm{Hz})\end{array}$ & $\begin{array}{c}\text { 'Maximum } \\
\text { Deformation }(\mu \mathrm{m})\end{array}$ \\
\hline single-beam & 1909.1 & 2780 & 161.64 & 225.05 \\
\hline dual-beam & 275.87 & 55.082 & 699.41 & 282.86 \\
\hline three beams & 267.44 & 29.757 & 1240.96 & 145.12 \\
\hline four beams & 119.54 & 21.071 & 1440.1 & 145.09 \\
\hline
\end{tabular}

As can be seen from Table 1, with the increase of the number of beams, stress and maximum deformation are significantly reduced, consistent with theoretical analysis results. When increasing the number of beams, the structure will become strong and the resonance frequency is constantly increasing. Contrary to this, the large deformation stress is relatively large, but collecting efficiency will limit and life will short. After a comprehensive consideration, selecting the four-beam structure as the final, but its natural frequency is not conducive to large low frequency vibration energy harvesting, it should be further optimized. 


\section{Optimization Process}

\section{Numerical Optimization}

The sensitivity of the maximum displacement of four-beam structure and the natural frequency with the beam's length, width, height is shown in Figure 5. We can read from the picture: the impact of the beam length on both are not large; beam width has the greatest impact on the maximum displacement, and with the increase of the width of the beam, the maximum displacement is reduced, the impact on the latter is after the height; like the length, height has little effect on the former, but the effect on the sensitivity of frequency is the highest. Alignment of frequency and the maximum displacement, we should be appropriate to increase the length, and reduce the width and height of the beam.

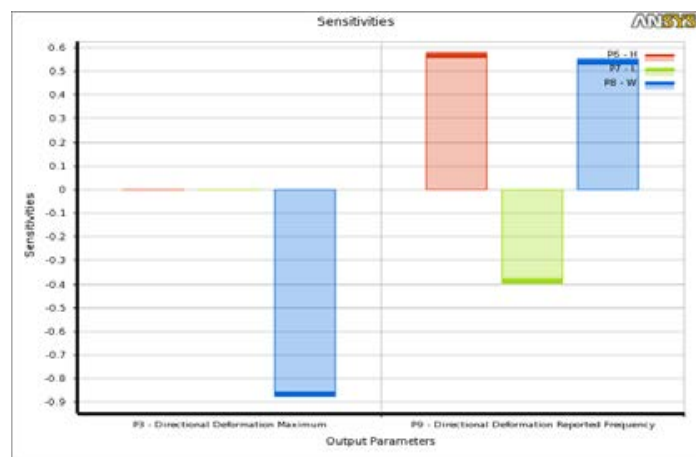

Fig. 5 The sensitivity of beam length, width and height to the maximum displacement and the frequency of the beam
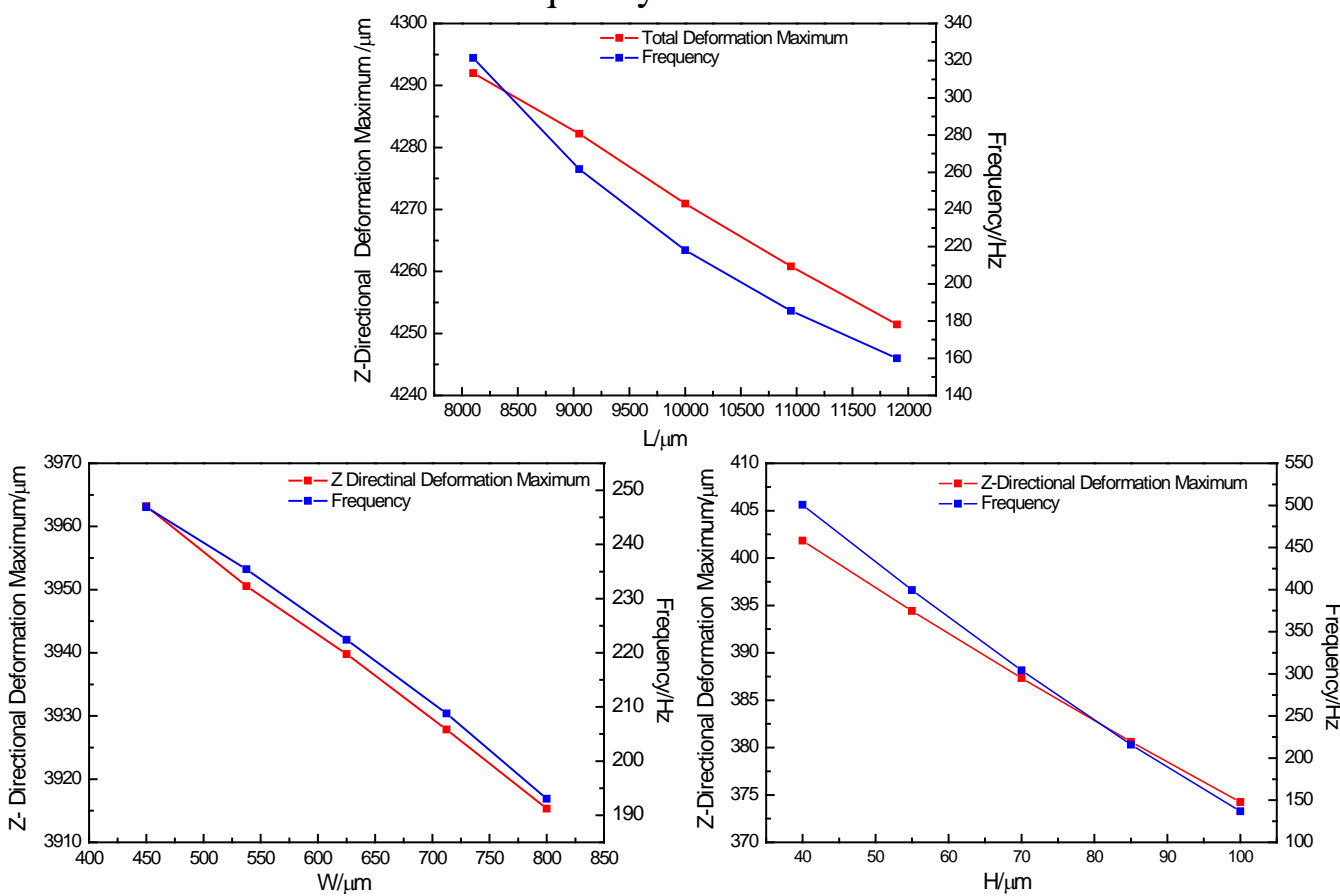

Fig. 6 The change of the maximum displacement of $\mathrm{Z}$ direction and the natural frequency with the structure parameter of the beam

Eventually, to meet the low frequencies $(100-300 \mathrm{~Hz})$ requirements, after the material selected, the length, width and height is set respectively $12000 \mu \mathrm{m}, 400 \mu \mathrm{m}, 40 \mu \mathrm{m}$, just getting the natural frequency $168.73 \mathrm{~Hz}$. Apparently the beam width beam height is reduced to the minimum, the beam length still reached $12000 \mu \mathrm{m}$, which does not apply to a limited narrow space.

\section{Structure Optimization}

Both to achieve low frequency and save space, the spiral structure can satisfy the requirements put forward, such a "straight to bend" to ensure that the length of the beam and realize the design goal. As shown in Figure 7 of the simulation results, the parameters are $L=7200 \mu \mathrm{m}, \mathrm{W}=500 \mu \mathrm{m}$, $\mathrm{H}=65 \mu \mathrm{m}$, and design the round corner instead of the right angle to avoid stress concentration. 


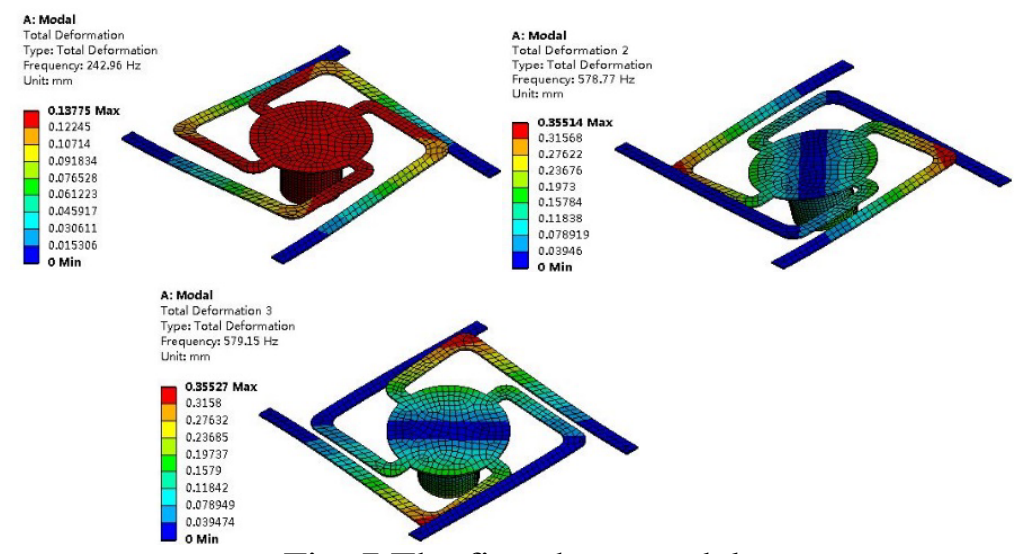

Fig. 7 The first three modal

The first six modal are obtained as shown in table 2,

Tab. 2 The frequency of the first six modal

\begin{tabular}{|l|l|l|l|l|l|l|}
\hline Model & $\begin{array}{l}\text { First } \\
\text { modal }\end{array}$ & $\begin{array}{l}\text { Second } \\
\text { modal }\end{array}$ & $\begin{array}{l}\text { Third } \\
\text { modal }\end{array}$ & $\begin{array}{l}\text { Fourth } \\
\text { modal }\end{array}$ & $\begin{array}{l}\text { Fifth } \\
\text { modal }\end{array}$ & $\begin{array}{l}\text { Sixth } \\
\text { modal }\end{array}$ \\
\hline Frequency $(\mathrm{Hz})$ & 242.9 & 578.8 & 579.2 & 2296.1 & 2395.8 & 2549.2 \\
\hline
\end{tabular}

Through the modal analysis, we can get the natural frequency of the vibration energy harvester, and know at which frequencies of the excitation, vibration energy harvester can reach maximum amplitude, which can get the best output electrical properties. But, on the other hand, the vibration energy of a particular structure, the device in the natural frequency of vibration, the risk of damage is also increased. So, the choice of excitation frequency, need to take into account two aspects, that is the output power characteristics and device life. By the modal analysis, the first modal is our desired vibration modal, other modal are interfering movement, we must try to suppress interference, so we will widen the gap between the interference signal and the first modal. Table 2 shows the frequency difference of the first modal and other fifth modal is great, which shows the cross coupling effect is small. While the other modal are relatively high, ensuring that the vibration energy collector is only moving in the sensitive direction when given a particular excitation frequency.

\section{Fatigue Analysis}

\section{Harmonic Response Analysis}

The frequency sweep range is set $50 \sim 450 \mathrm{~Hz}$, and the stress and acceleration changes of different frequencies are obtained in figure 8.

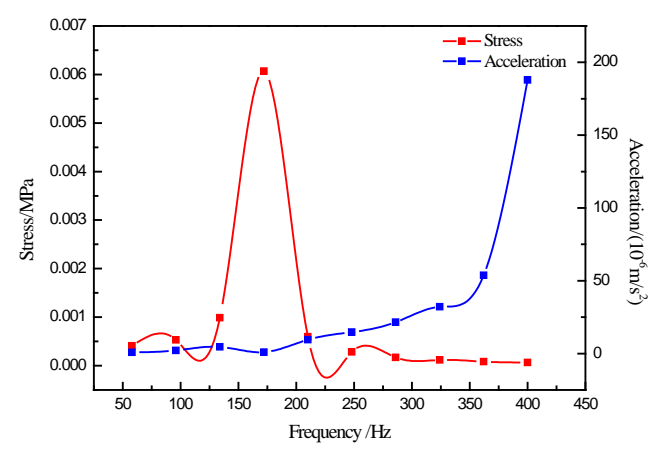

\section{Life Analysis}

Fig. 8 The acceleration and stress of different frequency

A practical energy harvester, the load received is random and dynamic, so that the analysis becomes more complex. In this case, it has been proved transient dynamic analysis in the time domain is almost impossible. In 1964 Bendat first proposed a request PSD (power spectral density) signal from the fatigue life of a method based on narrowband, 1985 Dirlik proposed for broadband signals experience fatigue analysis Closed Solutions, later Bishop achieved this proof [12]. 
ncode_designlife, with multi-file, multi-channel, multi-format features, is a mainstream fatigue analysis software, which has a wealth of time domain, frequency domain and statistical analysis functions.

From the above analysis, the energy harvester of the overall structure of the beam parameters will affect the stability and life of the entire system, so the beam parameter is set to $\mathrm{L}=7200 \mu \mathrm{m}$, $\mathrm{W}=500 \mu \mathrm{m}, \mathrm{H}=65 \mu \mathrm{m}$. Under constant acceleration shown in figure 9, its life time up $3.092 \times 10^{18}$, shown in figure 10, meeting the design requirements.

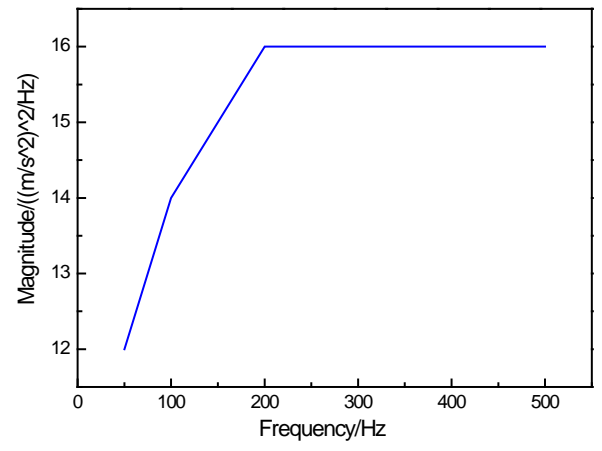

Fig. 9 Acceleration PSD for random excitation

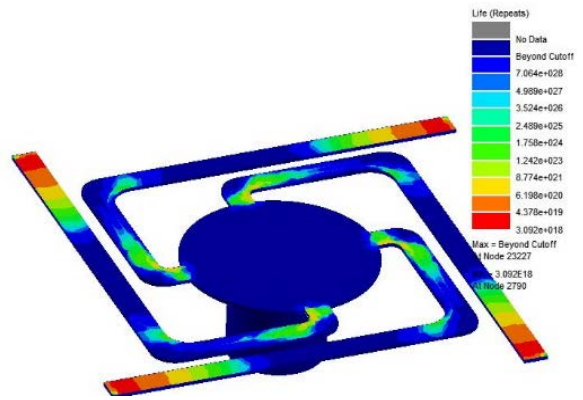

Fig. 10 The result of corresponding life distribution

\section{Summary}

The micro vibration energy harvester used in wireless power, can greatly enhance the independence and stability of wireless sensors. Through the above analysis, it is known that the influence of the geometric structure and geometrical parameters on the amplitude and frequency. Based on the finite element analysis method of ANSYS, the energy harvester with different shapes is simulated and analyzed, and the structure of the four beam structure is stable and the safety factor is high. Then the optimal parameters of the beam are obtained by optimizing the parameters of the four beam structure. Finally, the fatigue analysis of the energy collector is proved to meet the requirements of the fatigue life. On the one hand, this research provides some reference value for the design of micro vibration energy collector, on the one hand, it provides a description of the reliability of micro devices.

\section{Acknowledgments}

The authors appreciate the financial supported by the National Nature Science Foundation of China, Grant No.61401405 and 51475438.

\section{Reference}

[1] Bogue R. Energy harvesting and wireless sensors: a review of recent developments J. Sensor Review, 2009, 29(3):194-199.

[2] Moll, Francesc. Review of energy harvesting techniques and applications for microelectronics J. Proceedings of SPIE - The International Society for Optical Engineering 5837(2005):359-373.

[3] LEE S,YOUN B D,JUNG B C. Robust segment -type energy harvest and its application to 
wireless sensors J. SMART MATER STRUC. (2009) 18: 095021.

[4] Wang Peihong, Liu Huiting, Yang Zhuoqing, rt al. Sandwiched electromagnetic vibrations energy harvester based on MEMS technology J. NANOTECHNOL PRECIS ENG, (2010) 8(06):510-515.

[5] Vinod R Challa, M G Prasad and Frank T Fisher. Towards an autonomous self-tuning vibration energy harvesting device for wireless sensors network applications J. SMART MATER STRUC, (2011) 20(2); 025004.

[6] Information on http://www.mwrf.net/news/newtech/2015/18486.html.2015-12-25.

[7] Anderson, Todd A., and D. W. Sexton. A vibration energy harvesting sensors platform for increased industrial efficiency C // Proceedings of SPIE; SPIE. San Diego, CA, USA, 2006, 6174.

[8] Roundy, Shad, P. K. Wright, and J. Rabaey. J. A study of low level vibrations as a power source for wireless sensors nodes J. COMPUT COMMUN, 26.11(2003):1131-1144.

[9] Kuehne, Ingo, et al. "Power MEMS-A capacitive vibration-to-electrical energy converter with built-in voltage." Sensors \& Actuators A Physical 142.1(2008):263-269.

[10] Want, R., Farkas, K. I., \& Narayanaswami, C. (2005). Guest editors' introduction: energy harvesting and conservation. IEEE Pervasive Computing, 4(1), 14-17.

[11] Owens, Benjamin A. M., and B. P. Mann. "Linear and nonlinear electromagnetic coupling models in vibration-based energy harvesting." Journal of Sound \& Vibration 331.4(2012):922-937.

[12] Wang Guojun, Hu Renxi like like. nSoft Fatigue Analysis Theory and Applications M. Science Press, 2007: 236-258. 